\title{
Review of: "Dopaminergic medication increases motivation to exert cognitive control by reducing subjective effort costs in Parkinson's patients"
}

\author{
Corinna Kührt ${ }^{1}$ \\ 1 Technische Universität Dresden
}

Potential competing interests: The author(s) declared that no potential competing interests exist.

This preprint examines the moderating role of dopamine in the willingness to exert effortful control in Parkinson's disease. Healthy controls and Parkinson's patients were invited twice to perform a Demand Selection Task (DST) and several neuropsychological measures, while patients were either on or off their medication at the session. To test the hypotheses, in total nine mixed-effects models were calculated and interpreted. This bears the risk of overestimating the effects due to the cummulation of $\alpha$-error. The choice of predictors should be reconsidered.

I have some concerns with this preprint. The following aspects need to be addressed:

\section{Introduction}

The introduction introduces previous findings in a straightforward way and ends with the study design and hypotheses. The authors back-up their statements by several references. However, I wondered, whether all of these are truly relevant, e. g. when introducing the DST, it would be sufficient to cite the paper that first introduced this task. I recommend to go through the references and to keep only the most relevant ones to enhance readability and to facilitate traceability for the more interested reader.

\section{Material and methods}

1. To enhance transparency and reliability, it is helpful to provide all details of data collection and analysis. In this context, the 21-word solution (https://dx.doi.org/10.2139/ssrn.2160588) is a recommended statement and should be included if applicable.

2. This preprint is made available on the preprint server medRxiv. What about data, scripts and materials? Are they openly available, too? If yes, please add the repository. If no, why not?

\subsection{Participants}

1. Power and sample size estimations help researchers and readers to interpret the results. Was the sample size determined in advance and how? Does the resulting sample size have enough power to reveal the expected effects? 2. What were inclusion and exclusion criteria for patients? Were there additional inclusion or exclusion criteria for healthy controls (besides that they were age-matched and did not report "major health issues, neurological disorders, or active psychiatric problems")? 
3. How was participation compensated?

4. The authors state that six participants made $0 \%$ correct answers. I wondered whether these participants were able to differentiate between the colors in the paradigms that indicate the magnitude or parity judgment. Were participants asked or tested for color blindness or blue-yellow deficiency?

5. To what refers "see below" in "After examining response patterns in the DST, we found that six participants (4 Parkinson's patients and 2 healthy controls) evidenced difficulty understanding the task instructions, demonstrated by $0 \%$ correct responses in the final two instructed blocks of the DST (see below)."?

6. Table 1 displays the demographic information of the sample. To which statistical analysis do thep-values in the last column correspond?

\subsection{Procedure and design}

1. All participants were tested in two sessions with six weeks in between. How long was the duration of these sessions and each part, i. e. neurophysiological battery, task-switching paradigm, DST?

2. The participants went through a neuropsychological battery as well as two additional scales. As readers may not be familiar with these measures, it is beneficial to include a brief description and relevant psychometric data for each measure.

\subsection{Task-switching paradigm}

In the task-switching paradigm, participants made their response by pressing a button. For each response, reaction time was measured. The speed of such a response depends on handedness and the finger used to press the button; e. $g$. index finger is faster compared to middle finger. How did the authors control for that?

\subsection{Demand Selection Task (DST)}

Figure 1 gives the impression that the color patches were not centered on the screen. Is there a reason for that? Which locations were used for the patch presentation, except for the one displayed in Figure 1?

\subsection{Statistical Analysis}

1. Reaction times were preprocessed by including only correct answers and by log-transformation. Were there other preprocessing steps applied, like e. g. trimming?

2. As the SDMT-score significantly differed between healthy controls and Parkinson's patients, the authors considered this score to be involved into the statistical models. However, this score was only included, if it produced a significant main effect or differed between experimental groups. Even if it does not produce main effects in the models, all effects are controlled for this score. By this, an interference effect of this score can be excluded. Therefore, I recommend to keep the SDMT-score within all models.

\section{Results}

1. The introduction ends with the postulation of two hypotheses and one exploratory. These are: 
I) "[...] patients OFF dopamine would exhibit more effort-avoidant preferences, compared to when they were ON medication, as well as compared to healthy controls."

II) "In addition, we expected that patients ON medication would show similar rates of effort avoidance than healthy controls."

III) "Finally, the task-switching paradigm embedded in the DST allowed us to explore a possible relationship between participants' switch costs—as a proxy for individual effort costs—and effort-avoidant choice behavior in the DST." However, the results section seems to include some more analysis than I would expect, i.e. seven different mixed-effects models within the preprint and two more in the supplemental material. Therefore, I propose to state clearly a) which statistical test and model correspond to which hypothesis, b) which hypotheses are exploratory and c) to reconsider the statistical analysis applied. There are a lot of interaction effects tested, but no concrete hypothesis formulated. If the many analysis are to be retained, the results must be corrected for $\alpha$-error.

2. In addition, the statistical models applied are not appropriate to test a null-effect as it is postulated in hypotheses II. Null-effects are best tested with equivalence tests.

3. Tables are a good way to display results from mixed-effects models. The standard deviation of the random effects are important to interpret the results and, therefore, should be provided.

4. The interpretation of results presented in Figures 2, 3 and 4 can be facilitated, if significant results were labeled as such.

\subsection{Dopaminergic medication reduces effort avoidance in Parkinson's patients}

1. This section starts with a descriptive evaluation of the hypotheses that dopamine reduces effort-avoidant preferences and compares groups using one-sample t-tests. When interaction effects are evaluated, as it is the case here, estimated marginal means are preferred over observed group means. The latter do not represent the interaction data, i.e. they include both the grand mean, the main effects means and the interaction all together. In contrast, estimated marginal means additionally account for all predictors in the model. Therefore, a simple slopes analysis basing on the mixed effects model is the preferred test for interactions. It allows also creating figures with estimated marginal means that display interpretable effects. The same applies to the descriptive evaluation in section 3.2.

2. Mixed-effects model reported in Table 4 is an extension of the model reported in Table 2 by the variable detection ability. Both models answer the same research question. $\alpha$-error needs to be corrected in order to avoid $\alpha$-error cummulation. A better option is to exclude the redundant model. The same applies to the two models reported in Table 6 and the additional model mentioned in 3.3. Before applying a statistic, the parameters (i.e. predictors) should be well selected and literature-guided.

3. Which hypothesis answers the additional model reported in the supplemental material? The main result should be backed-up by statistics.

\subsection{Differences in choice behavior are not explained by task-switching performance}




\subsection{Individual differences in switch costs do not predict effort avoidance in Parkinson's disease}

1. The mixed-effects model revealed a disease state $\mathrm{x}$ detection ability interaction with $p=.050$ and .069 , respectively. This result must be interpreted as nonsignificant as $p$-values are not below .050. Moreover, this interaction is not displayed in Table 6., but the interaction between disease state and switch costs (RT) yielding the reported results.

2. In the end of this section, the authors refer to an additional analysis. Which hypothesis is tested by this analysis? If these predictors are reasonable basing on the literature, this should be explained in the preprint and these predictors should be included into the main model.

\section{Discussion}

1. The authors discuss their findings comprehensively and compare them with other studies.

2. The paragraph discussing the exploratory findings, i.e. disease state $x$ detection ability interaction, needs revision, as the results are not significant.

3. The last paragraph contains the conclusion. The authors should take care to interpret the results in terms of Parkinson's disease.

\section{Formal issues}

1. I recommend the indication of page numbers and line numbers. It highly facilitates reviewing and referencing.

2. The headings are inconsistently in sentence or upper case.

3. The tables should have headings that appear above the table.

In summary, it is an interesting preprint that contributes to the prevailing literature, but requires revision of methodological aspects. 